\title{
TWO EMBEDDING THEOREMS FOR FINITE LATTICES
}

\author{
JURIS HARTMANIS
}

Introduction. One of the important unsolved problems of lattice theory is the problem of embedding every finite lattice in a finite partition lattice. The partition lattice can be considered as the lattice of subspaces of a suitable geometry. By slightly loosening the conditions on this geometry it will be shown that every finite lattice can be embedded in the lattice of subspaces of a finite geometry. Using this result it will be shown that every finite lattice can be embedded in the lattice of all geometries on a finite set. The lattice of all geometries will be shown to be again the lattice of subspaces of a geometry and it will be seen that its structure is similar to that of a partition lattice. This reduces the above mentioned problem to the problem of embedding every finite lattice of geometries in a finite partition lattice.

1. Preliminaries. Throughout this paper the term geometry will be used in the following sense.

Definition 1. A geometry $G$ on a set $S$ is a collection of subsets of $S$, such that any two distinct elements of $S$ are contained in one and only one subset and every subset contains at least two distinct elements.

The elements of the set $S$ are called points and the subsets of Definition 1 are called lines of the geometry $G$. A line is said to be trivial if it contains only two distinct points, nontrivial otherwise. A subset $T$ of $S$ is said to be a subspace of $G$ if with any two distinct points of $T$ the line defined by these points is contained in $T$.

It can easily be shown that the set of all subspaces of a geometry $G$ forms a complete point lattice under set inclusion. We shall call it the lattice of the geometry $G$.

Let $G$ and $H$ be two geometries on the set $S$. We shall say that $G \geqq H$, if for every line $l$ in $H$ there exists a line $L$ in $G$ such that $l \subseteq L$. It can be shown that the collection of all geometries on a set $S$ forms a complete point lattice under the above defined ordering. We shall denote the lattice of all geometries on the set $S$ by $L G(S)$. From Definition 1 it is seen that we can consider a geometry as a generalized partition. It is also easily seen that the set of geometries on a set $S$ whose nontrivial lines all contain a fixed point form a sublattice of $L G(S)$ and that this sublattice is isomorphic to a partition lattice.

Presented to the Society, October 22, 1955; received by the editors August 3, 1955. 
THEOREM 1. $L G(S)$ is isomorphic to the lattice of subspaces of some geometry.

Proof. The points of $L G(S)$ are geometries with only one nontrivial line and this line consists of three points. We shall denote the point $P$ of $L G(S)$, whose nontrivial line consists of $x, y, z$, by $\{(x, y, z)\}$. The union of any two distinct points $P$ and $R$ is either a geometry with only one nontrivial line and this line consists of four points or a geometry with two nontrivial lines and these lines contain three points each. For either case $P \cup R$ covers $P$ and $R$. This implies that the subsets of points of $L G(S)$ which are contained in unions of two distinct points form the set of lines for some geometry on the set of points of $L G(S)$. Thus we just have to show that $L G(S)$ is the lattice of subspaces of this geometry. To do this we have to show that if $T$ is a subspace of this geometry and the point $\{(a, b, c)\} \leqq U T$ $=\bigcup\{P \mid P \in T\}$ in $L G(S)$, then $\{(a, b, c)\} \in T$. The elements $a, b, c$ are contained in some line $L$ of the geometry $U T$. Let $N$ be the set of points $\{(x, y, z)\}$ such that $x, y, z$ are contained in $L . N$ is a subspace of the above defined geometry since $N$ is the set of points contained in an element of $L G(S)$. This element is the geometry with the only nontrivial line $L$ and we shall denote it by $\{L\}$. Let $T_{N}=T \wedge N$. $T_{N}$ is a subspace and it can be seen that $U T_{N}=\{L\}$. To complete the proof we shall construct an auxiliary geometry $G$ on the set of points of $L G(S)$. To do this let us consider the subsets $U$ of $S$ with the property that if the distinct elements $x, y, z$ are contained in $U$ then the point $\{(x, y, z)\} \in T_{N}$. Let us denote the collection of all such subsets by $\mathfrak{F}$. Let $l(p, q)$ denote the line of $G$ which contains $p$ and $q$. Let $l(p, q)$ contain only $p$ and $q$ if there is no set $U$ in $\mathfrak{F}$ which contains $p$ and $q$, otherwise let $l(p, q)=V\{U \in \mathfrak{F} \mid p, q \in U\}$. Since $T_{N}$ is a subspace we can show that the nontrivial lines $l(p, q)$ are maximal elements of the set $\mathfrak{F}$. This implies that any two distinct lines of $G$ can have at most one point in common. Thus $G$ is a geometry. From the definition of $G$ we see that if $\{(x, y, z)\} \leqq G$ then $\{(x, y, z)\} \in T_{N}$. On the other hand, if $\{(x, y, z)\} \in T_{N}$ then the set consisting of $x, y, z$ is an element of $\mathfrak{F}$ and therefore $\{(x, y, z)\} \leqq G$. Thus $U T_{N}=G=\{L\}$ and therefore $\{(a, b, c)\} \leqq U T$ implies that $\{(a, b, c)\} \in T$. This completes the proof.

\section{Embedding theorems.}

THEOREM 2. Every finite point lattice can be embedded in the lattice of some finite geometry.

PROOF. Let $L$ be a finite point lattice with the points $p_{1}, p_{2}, \cdots, p_{N}$. 
We shall construct a geometry $G$ on a finite set $S$ such that $L$ is isomorphic to a sublattice of the lattice $L^{\prime}$ of this geometry. To do this let $S_{1}, S_{2}, \cdots, S_{N}$ be the nontrivial lines of $G$ and let these lines be disjoint. For $i=1,2, \cdots, N$ let $\theta$ map $p_{i}$ of $L$ on to the line $S_{i}$ of $G$. In general we shall add other nontrivial lines to $G$ in order to preserve the unions and intersections under the mapping $\theta$. We shall now describe the construction of the additional nontrivial lines. Assume

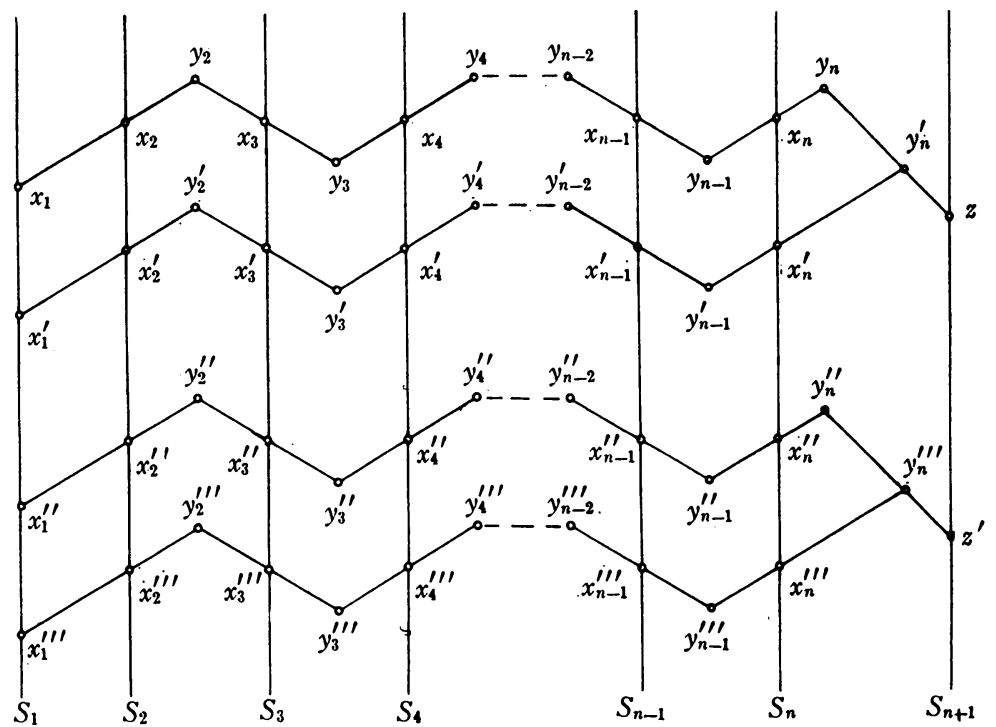

FIG. 1

that, after re-enumerating if necessary, the union of the points $p_{1}, p_{2}, \cdots, p_{n}$ of $L$ contains the point $p_{n+1}$ and that no union of a smaller number of the points $p_{1}, p_{2}, \cdots, p_{n}$ contains $p_{n+1}$. The additional nontrivial lines of $G$ will be so constructed that the corresponding union of $S_{1}, S_{2}, \cdots, S_{n}$ in $L^{\prime}$ contains $S_{n+1}$ and that no union of a smaller number of the lines $S_{1}, S_{2}, \cdots, S_{n}$ does so. To do this let the distinct points $x_{i}, x_{i}^{\prime}, x_{i}^{\prime \prime}, x_{i}^{\prime \prime \prime}$ be contained in $S_{i}$ for $1 \leqq i \leqq n$, let $z, z^{\prime}$ be contained in $S_{n+1}$ and let $y_{i}, y_{i}^{\prime}, y_{i}^{\prime \prime}, y_{i}^{\prime \prime \prime}, 2 \leqq i \leqq n$, be contained in a subset $R$ of $S$ which is disjoint from $S_{1}, S_{2}, \cdots, S_{N}$. Let none of these points be contained in any other construction of additional nontrivial lines. Then we shall add to $G$ the lines $\left(x_{1}, x_{2}, y_{2}\right),\left(y_{2}, x_{3}, y_{3}\right)$, $\left(y_{3}, x_{4}, y_{4}\right), \cdots,\left(y_{n-1}, x_{n}, y_{n}\right)$, the corresponding lines in the primed, double primed, and triple primed elements and finally the lines $\left(y_{n}, y_{n}^{\prime}, z\right)$ and $\left(y_{n}^{\prime \prime}, y_{n}^{\prime \prime \prime}, z^{\prime}\right)$. This construction is shown in Figure 1. We see that any two of these nontrivial lines have at most one point 
in common and since all the different constructions of additional nontrivial lines will be done on disjoint sets of points $G$ will be a geometry. We also see that any subspace $T$ of $G$ which contains the first $n$ lines $S_{1}, S_{2}, \cdots, S_{n}$ of this construction will contain the points $z, z^{\prime}$ and therefore $T$ will contain the last line $S_{n+1}$ of this construction. Let us assume that we have adjoined the necessary nontrivial lines for all the unions as described above. We shall now characterize the union $\bigcup\left\{S_{i} \mid i \in A\right\}$ in $L^{\prime}$. Let $\bigcup\left\{p_{i} \in L \mid i \in A\right\}=a$ be the corresponding union in $L$ and let $\bar{A}=\left\{i \mid p_{i} \leqq a\right\}$. With the index set $\bar{A}$ we associate a set $P_{\bar{A}}$ which is a subset of the elements of $R$ which are used to construct additional nontrivial lines. To determine whether such a point $y$ is contained in $P_{\bar{A}}$ let us re-enumerate the lines and points of the construction of additional lines in which $y$ is contained as we did in Figure 1. Then $y=y_{k}$ and $y_{k}$ is contained in $P_{\bar{A}}$ if and only if the first $k$ lines $S_{1}, S_{2}, \cdots, S_{k}$ of this construction are contained in the set $\left\{S_{i} \mid i \in \bar{A}\right\}$. Let us denote the set $\bigvee\left\{S_{i} \mid i \in \bar{A}\right\}$ $\vee P_{\bar{A}}$ by $T$. We can show that $T$ is a subspace of $G$ and that $\bigvee\left\{S_{i} \mid i \in A\right\}$ $\subseteq T \subseteq \cup\left\{S_{i} \mid i \in A\right\}$. This implies that $\cup\left\{S_{i} \mid i \in A\right\}=\bigvee\left\{S_{i} \mid i \in \bar{A}\right\}$ $\bigvee P_{\bar{A}}$. From this we see that $p_{j} \leqq \bigcup\left\{p_{i} \in L \mid i \in A\right\}$ if and only if $S_{j} \leqq \cup\left\{S_{i} \mid i \in A\right\}$, which shows that $\theta$ defines a one-to-one order preserving mapping of $L$ onto the set of elements of $L^{\prime}$ which are unions of the elements of $\left\{S_{1}, S_{2}, \cdots, S_{N}\right\}$. We shall now show that these unions are closed under intersections and therefore form a sublattice of $L^{\prime}$ which is isomorphic to $L$. Let $\vee\left\{S_{i} \mid i \in \bar{A}\right\} \vee P_{\bar{A}}$ and $\vee\left\{S_{i} \mid i \in \bar{B}\right\} \vee P_{\bar{B}}$ be two unions and let $\bar{A} \wedge \bar{B}=\bar{C}$. We know that the sets $P_{\bar{A}}$ and $P_{\bar{B}}$ are disjoint from the sets $S_{1}, S_{2}, \cdots, S_{N}$. From the definition of the sets $P_{\bar{A}}$ and $P_{\bar{B}}$ it follows that $P_{\bar{A}} \wedge P_{\bar{B}}=P_{\bar{C}}$. Thus the intersection of these unions is given by $\vee\left\{S_{i} \mid i \in \bar{C}\right\} \vee P_{\bar{c}}$, which shows that they are closed under intersection. This completes the proof.

We know that every finite lattice can be embedded in a finite point lattice. Thus by Theorem 2 we obtain the more general result.

CoRollary 1. Every finite lattice can be embedded in the lattice of a finite geometry.

Lemma 1. Let $L$ be the lattice of a geometry $G$ on a finite set $S$. Then $L$ can be embedded in the lattice $L^{\prime}$ of the geometry $G^{\prime}$ on a finite set $S^{\prime}$ such that every point of $G^{\prime}$ is contained in at least one nontrivial line.

Proof. For every point $p_{i}$ of $G$ which is not contained in a nontrivial line of $G$ we shall add two new points $p_{i}^{\prime}, p_{i}^{\prime \prime}$ to $S$ and a new line consisting of $p_{i}, p_{i}^{\prime}$ and $p_{i}^{\prime \prime}$ to $G$. Let this be the geometry $G^{\prime}$ on $S^{\prime}$ and denote its lattice by $L^{\prime}$. Every subspace $T$ of $G$ is a subspace 
of $G^{\prime}$ and the set of all subspaces of $G$ is closed under intersection and union in $G^{\prime}$. Thus $L$ is a sublattice of $L^{\prime}$.

THEOREM 3. Let $L$ be the lattice of a geometry $G$ on a finite set $S$. Then $L$ can be embedded in the lattice of all geometries on some finite set $S^{\prime}$.

Proof. Let $G$ be a geometry with the points $p_{1}, p_{2}, \cdots, p_{m}$ and with the nontrivial lines $l_{1}, l_{2}, \cdots, l_{n}$. By Lemma 1 we may assume that every point of $G$ is contained in at least one nontrivial line. We shall map every point $p_{i}$ of $G$ onto a geometry $G_{i}$ on $S^{\prime}$ and show that these geometries generate a sublattice of $L G\left(S^{\prime}\right)$ which is isomorphic to $L$. Let us denote this mapping by $\theta$. To construct the geometry $G_{i}$ we shall define certain subsets of $S^{\prime}$ which will be used in this construction. Let $R, S_{0}, S_{1}, S_{2}, \cdots, S_{n}$ be disjoint subsets of $S^{\prime}{ }^{1}$ With every point $p_{j}$ of $G$ we associate a set $A_{j}$ which is contained in the set union of $S_{1}, S_{2}, \cdots, S_{n}$ and is such that $A_{j}$ has exactly one point in common with the set $S_{i}$ if the point $p_{j}$ is on the line $l_{i}$ of $G$ and has no points in common with $S_{i}$ otherwise. Let any two such sets $A_{i}$ and $A_{j}$ be disjoint if they are associated with distinct points $p_{i}$ and $p_{j}$, respectively. Let $l_{k}$ be a nontrivial line of $G$ and let, after reenumerating if necessary, $p_{1}, p_{2}, \cdots, p_{t}$ be the points of this line. Then we define $L_{k}=A_{1} \bigvee A_{2} \bigvee \cdots \vee A_{t}-S_{k}$. With every pair $\left(a, L_{k}\right), a \in L_{k}$, we associate the three subsets each consisting of three points: $\pi_{1}=\left(x_{0}, x_{k}, z\right), \pi_{2}=\left(x_{0}^{\prime}, x_{k}^{\prime}, z^{\prime}\right)$ and $\pi_{3}=\left(z, z^{\prime}, a\right)$. Let the distinct points $x_{0}, x_{0}^{\prime}$ be contained in $S_{0}$, let $x_{k}, x_{k}^{\prime}$ be contained in $S_{k}$, and let $z, z^{\prime}$ be contained in $R$. Let none of these points except $a$ be contained in $A_{i}, i=1,2, \cdots, m$. Let two such triplets of subsets which are associated with $\left(a, L_{k}\right)$ and $\left(b, L_{j}\right)$, respectively, have no points in common if $a \neq b$ and only the point $a$ in common if $a=b$, $k \neq j$. We shall denote the set of all such subsets $\pi_{i}$ by $P . Z_{k}$ shall denote the set consisting of points $z, z^{\prime}$ of $R$ which are contained in some set $\pi_{i}$ associated with $\left(a, L_{k}\right), a \in L_{k}$.

To construct the geometry $G_{i}$ on $S^{\prime}$ we let the set of nontrivial lines of $G_{i}$ consist of $S_{0} \vee A_{i}, S_{1}, S_{2}, \cdots, S_{n}$ and all the elements $\pi_{i}$ of $P$. Any two of these nontrivial lines have at most one point in common which shows that it is a set of nontrivial lines of some geometry on $S^{\prime}$. Since every point $p_{i}$ of $G$ is contained in at least one nontrivial line of $G$ the set $A_{i}, i=1,2, \cdots, m$, is nonvoid. Since these sets are disjoint we see that the geometries $G_{1}, G_{2}, \cdots, G_{m}$ are noncomparable in $L G\left(S^{\prime}\right)$. Thus the mapping $\theta$ is a one-to-one mapping of the

\footnotetext{
${ }^{1}$ It can be shown that $S^{\prime}$ has to contain at most $m n(6 n+1)$ points, $R$ and $S_{0}$ at most $2 m n$ points each and $S_{i}, i=1,2, \cdots, n$, at most $m(2 n+1)$ points.
} 
set of points of $L$ onto the set consisting of the geometries $G_{1}, G_{2}, \cdots$, $G_{m}$. We also see that the intersection of any two such distinct geometries $G_{i}$ and $G_{j}$ is the geometry whose set of nontrivial lines consists of $S_{0}, S_{1}, S_{2}, \cdots, S_{n}$ and all the elements $\pi_{i}$ of $P$.

If $p_{i}$ and $p_{j}$ are distinct points of $G$ which are not contained in the same nontrivial line then $G_{i} \cup G_{j}=E$ in $L G\left(S^{\prime}\right)$ and the set of nontrivial lines of $E$ consists of $S_{0} \vee A_{i} \vee A_{j}, S_{1}, S_{2}, \cdots, S_{n}$ and all the elements $\pi_{i}$ of $P$. First, we shall show that any two of these nontrivial lines have at most one point in common. The line $S_{0} \vee A_{i} \vee A_{j}$ can have at most one point in common with the line $S_{k}$ since otherwise $A_{i}$ and $A_{j}$ would have points in common with the set $S_{k}$ which would imply that the points $p_{i}$ and $p_{j}$ are contained in the nontrivial line $l_{k}$ of $G$, contrary to assumption. From the definition of the line $\pi_{k}$ contained in $P$ it follows that $\pi_{k}$ can have at most one point in common with $S_{0} \vee A_{i} \vee A_{j}$ and $S_{i}, i=1,2, \cdots, n$. Thus $E$ is a geometry. We see that every line of $G_{i}$ and $G_{j}$ is contained in a line of $E$, thus $G_{i}, G_{j} \leqq E$. Since $S_{0} \vee A_{i}$ and $S_{0} \vee A_{j}$ are lines of $G_{i}$ and $G_{j}$, respectively, and they have more than one point in common $S_{0} \vee A_{i} \vee A_{j}$ is contained in a line of $G_{i} \cup G_{j}$, but then every line of $E$ is contained in a line of the union and therefore $E \leqq G_{i} \cup G_{j}$. From this it follows that $E=G_{i} \cup G_{j}$. Similarly we can show that if $p_{i}$ and $p_{j}$ are distinct points of $G$ which are contained in the nontrivial line $l_{k}$ then $G_{i} \cup G_{j}$ $=E$ and the set of nontrivial lines of $E$ consists of $S_{0} \bigvee S_{k} \bigvee L_{k} \bigvee Z_{k}$, $S_{1}, S_{2}, \cdots, S_{k-1}, S_{k+1}, \cdots, S_{n}$ and all the elements $\pi_{i}$ of $P$ which are not associated with a pair $\left(a, L_{k}\right), a \in L_{k}$.

From these results it follows that $p_{k} \leqq p_{i} \cup p_{j}$ in $L$ if and only if $G_{k} \leqq G_{i} \cup G_{j}$ in $L G\left(S^{\prime}\right)$. Thus these unions define a set of lines on the set consisting of $G_{1}, G_{2}, \cdots, G_{m}$, and these lines are preserved under the mapping $\theta$. To complete the proof we shall show that the geometries $G_{1}, G_{2}, \cdots, G_{m}$ generate a sublattice of $L G\left(S^{\prime}\right)$ and that this sublattice is the lattice of the above defined geometry on $\left\{G_{1}\right.$, $\left.G_{2}, \cdots, G_{m}\right\}$. Let $T=\left\{p_{i} \in L \mid i \in B\right\}$ be a subspace of $G$ and let $\left\{G_{i} \mid i \in B\right\}$ be the corresponding subspace in the geometry on $\left\{G_{1}, G_{2}, \cdots, G_{m}\right\}$. Let, after re-enumerating if necessary, $l_{1}, l_{2}, \cdots, l_{t}$ be the nontrivial lines which are contained in $T$ and let $p_{1}, p_{2}, \cdots, p_{s}$ be the points of $T$ which are not contained in nontrivial lines in $T$. Then $U\left\{G_{i} \mid i \in B\right\}=H$ and the set of nontrivial lines of the geometry $H$ consists of $\left[S_{0} \vee S_{1} \vee S_{2} \vee \cdots \vee S_{t} \vee Z_{1} \vee Z_{2} \vee \cdots \vee Z_{t}\right]$ $\bigvee\left[\bigvee\left\{A_{i} \mid i \in B\right\}\right], S_{t+1}, S_{t+2}, \cdots, S_{n}$ and all the elements $\pi_{i}$ of $P$ which are not associated with $\left(a, L_{i}\right), a \in L_{i}, i=1,2, \cdots, t$. Exactly as in the two previous cases we show that any two nontrivial lines can have at most one point in common and that $H=\cup\left\{G_{i} \mid i \in B\right\}$. 
From this follows that $G_{j} \leqq H$ if and only if $G_{j}$ is contained in the subspace $\left\{G_{i} \mid i \in B\right\}$. Thus to complete the proof we need only to show that these unions are closed under intersections. A straightforward but somewhat lengthy computation shows that if $T_{1}$ and $T_{2}$ are subspaces of the above defined geometry on the set consisting of $G_{1}$, $G_{2}, \cdots, G_{m}$, then $\mathrm{U}\left(T_{1}\right) \wedge \mathrm{U}\left(T_{2}\right)=\bigcup\left(T_{1} \wedge T_{2}\right)$. This shows that $G_{1}, G_{2}, \cdots, G_{m}$ generate a sublattice of $L G\left(S^{\prime}\right)$ which is isomorphic to $L$. This completes the proof.

From Theorem 2 and Theorem 3 we obtain the final result.

Theorem 4. Any finite lattice can be embedded in the lattice of geometries on some finite set.

\section{REFERENCES}

1. Oystein Ore, Theory of equivalence relations, Duke Math. J. vol. 9 (1942) pp. 573-627.

2. G. Birkhoff, Lattice theory, New York, 1948.

California Institute of Technology 